\title{
Pitch and Lengthening as Cues to Turn Transition in Swedish
}

\author{
Margaret Zellers \\ Institutionen för Tal, Musik \& Hörsel, Kungliga Tekniska Högskolan, Stockholm, Sweden \\ zellers@kth.se
}

\begin{abstract}
In many cases of turn transition in conversation, a new speaker may respond to phonetic cues from the end of the prior turn, including variation in prosodic features such as pitch and final lengthening. Although consistent pitch and lengthening features are well-established for some languages at potential points of turn transition, this is not necessarily the case for Swedish. The current study uses a two-alternative forced choice task to investigate how variation in pitch contour and lengthening at the ends of syntactically complete turns can influence listeners' expectations of turn hold or turn transition. Both lengthening and pitch contour features were found to influence listeners' judgments about whether turn transition would occur, with shorter length and higher final pitch peaks associated with turn hold. Furthermore, listeners were more certain about their judgments when asked about turn-hold rather than turn-change, suggesting an imbalance in the strength of turn-hold versus turn-transition cues.

Index Terms: prosody, perception, turn-taking
\end{abstract}

\section{Introduction}

The apparent ease with which transition from one speaker to another occurs in conversation has been well-established ever since Sacks, Schegloff \& Jefferson's [1] seminal paper, although recent work has indicated that these transitions are not as smooth as Sacks et al. originally proposed. Heldner \& Edlund [2] found a large degree of variation in timing of the onsets of new speakers' turns, with the greatest number of turn transitions across three corpora having a "just-noticeable" gap (i.e. silence) of around 200ms. Heldner [3] showed further that overlaps and gaps at turn transitions must have a duration of at least $120 \mathrm{~ms}$ in order to be perceptible to listeners. [2] argue that on the basis of their data, at least $41 \%$ of cases had a long enough gap that the next speaker could potentially use phonetic information from the end of the prior turn as a cue to turn transition.

In English, intonational patterns at prosodic boundaries have been associated with cuing turn hold or transition. Local, Kelly \& Wells [4] note that high pitch rises or low falls generally occur at speaker transition in Tyneside English, along with other phonetic features such as slowing and vowel centralization. This intonational finding has recently been replicated by Gravano \& Hirschberg [5] in a corpus of American English. Similarly, Auer [6] reports that "integrated" pitch contours, which do not display characteristics drastically different from what has come before, are often used in turn-continuations in German. However, in Central Swedish, the case is somewhat more complicated, as words carry obligatory contrastive pitch accents, and the final pitch accent often carries an additional $\mathrm{H}$ tone as a focus marker (cf. Gårding [7]; Gussenhoven \& Bruce [8]). House [9], [10] found that raising fundamental frequency (F0) and moving the focal F0 peak to the right led Swedish listeners to interpret utterances as questions. Furthermore,
House [10] demonstrated that rising intonation, while acceptable as a means of marking questions in Swedish, occurs in only $22 \%$ of cases, with children more likely than adults to use this type of marking. This suggests that manipulation of boundary material is not a preferred method of signaling meaning in Swedish, even if it is permissible.

Hjalmarsson [11] investigated the influence of final intonation patterns and final lengthening, as well as other cues such as semantic completeness, cue phrases, and phonetic features such as plosive release, on listeners' judgments about which speaker was going to continue in a Swedish recorded dialogue. She found that all of these features, except degree of final lengthening, had an influence on listeners' judgments about whether turn transition would occur, and that the presence of multiple, matching cues strengthened this influence. In her study, "flat" intonation was associated with turn-holding, while "falling" intonation was associated with turn-yielding. This is consistent with findings by Edlund \& Heldner [12], who also found that rising intonation patterns were not consistently associated with either turn-holding or turn-yielding. Although [11] did not find that listeners made consistent predictions of turn-transition on the basis of final lengthening, Hjalmarsson \& Laskowski [13] showed that including final lengthening in an automatic model improved prediction of speaker change at pauses, with increased final lengthening before the pause being associated with turn hold.

These somewhat contradictory findings indicate that there is more work to be done to understand prosodic cues associated with turn transition in Swedish. Previous studies using data from corpora have not been able to control for other sources of variation. In particular, syntactic completion or non-completion has been shown to be stronger than prosodic cues in predicting turn continuation (Auer [6]; Wichmann \& Caspers [14]), despite the fact that cues combine to create a gestalt (Ford, Fox \& Thompson [15]). Eliminating some of this kind of variation from the data can thus be a valuable tool to investigate the strength of prosodic cues. The current study explores the role of pitch and length variation in cueing turn transition, using a set of controlled stimuli in a perception paradigm.

\section{Experimental design}

\subsection{Stimuli}

The stimuli for this experiment were conversational turns drawn from the same set used by Hjalmarsson [11], taken from the DEAL corpus (Hjalmarsson et al. [16]), in which participants role-played bargaining at a flea market. The turns chosen for the current experiment had to be recognizably syntactically complete, with an accent on the final word but not the final syllable, and with a pitch contour consistent with the presence of a final focal accent (i.e. an F0 peak). In addition, for purposes of the pitch resynthesis, the segmental structure in the final word was restricted as much as possible 
to voiced segments. Four turns were finally chosen as the base tokens for the analysis:

- Designen är svensk men tillverkningen [är] i Kina. (The design is Swedish but the manufacturing [is done] in China.)

- Nej, jag har en lite större. (No, I have a slightly bigger one.)

- Så blir det lite svårare. (In that case it gets a little harder.)

- Ja jag har tittat lite på ahm på den där sågen. (Yes I’ve looked a little at uhm at that saw.)

The first three turns (Kina, större, svårare) were produced by the same male speaker, while the fourth (sågen) was produced by a female speaker.

\subsubsection{Resynthesis}

Twenty-six versions of each sentence were created using PSOLA resynthesis in Praat [17]. Of these, 14 were used for the current study; these will be described here. First, two Length versions were created. In the Short condition, final rhyme segments were normalized to a length of 0.1 seconds; in the Long condition, they were normalized to a length of 0.2 seconds.

Each Length version then had a number of pitch contours superimposed. The pitch contours involved two kinds of variation. The Peak set had the final F0 peak of the turn set at 3 semitones (st), 5st, and 8 st above the speaker's baseline. The 8st movement only occurred in the Long stimuli in order to respect the limits of the human articulatory system (cf. Xu \& Sun [18]). The Truncation set had the final low pitch either at the speaker's baseline, or at 2st, 4st, or 6st above it; Truncation values had to be lower than the pitch peak; so, for example, 6st truncation only occurred with an 8st peak.

In order to conduct the pitch resynthesis, three pitch points were set: at the onset of the stressed syllable rhyme $(\mathrm{V}=$ valley); at the location of the pitch peak in the original utterance $(\mathrm{P}=$ peak); and at the end of phonation ( $\mathrm{F}=$ final). In all cases, $\mathrm{V}$ was set equal to the lowest non-final pitch measured in the turn. The heights of $\mathrm{P}$ and $\mathrm{F}$ were then varied using a Praat script, as illustrated in Figure 1.
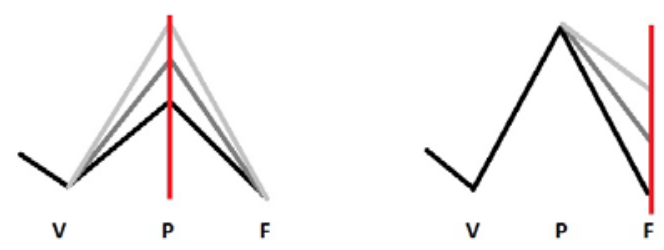

Figure 1: Pitch manipulations. Left: height manipulations (3st, 5st, 8st). Right: truncation manipulations (number varies for different pitch heights). $V=$ valley; $P=$ peak; $F=$ final.

\subsubsection{Naturalness ratings}

Since the stimuli were resynthesized, it was necessary to test whether the resynthesis was detectable and whether the resulting tokens were acceptable tokens in Swedish. In order to test this, 5 native speakers of Swedish listened to all of the resynthesized stimuli in randomized order and rated each token from 1 (very unnatural) to 4 (very natural). Since an exactly neutral rating would be 2.5 , in the main experiment, only tokens which had a mean rating of 2.66 or higher were used. After excluding tokens which did not reach this threshold, 50 of the original 56 stimuli remained.

\subsection{Methodology}

The experiment used a two-alternative forced-choice methodology, and was presented using Praat MFC. Pairs of stimuli were presented that varied in length, peak height, truncation amount, or some combination of these factors; the base sentence for each pair was always the same (so participants heard two versions of e.g. the större case, rather than one större and one svårare). Since the 50 stimuli were not evenly balanced across the sentences, the total number of comparisons that could be made was 101 .

Four versions of the experiment were created. There were two question conditions: in the Hold case, participants were asked to select the version of the sentence that the speaker would say if s/he wanted to continue speaking, while in the Change version they were asked to select the version the speaker would say if $\mathrm{s} / \mathrm{he}$ was done speaking. In each condition, there was a version in which the stimuli were presented in the order $A B$, and one in which the stimuli were presented in the order BA; this was done in order to eliminate any possible bias resulting from the order of presentation. The order of presentation was automatically randomized for each participant by Praat. Participants could re-listen to the stimuli pairs up to twice if they chose, but after initial acclimation to the task, most listened to each pair only once. After making their choice between the two stimuli, participants were also asked to state whether they were fairly sure or fairly unsure about their decision.

Eighteen native speakers of Swedish took part in the experiment (ages 20-68, median 25; 12 female). Participants were tested in a quiet room either at the Department of Speech, Music \& Hearing, KTH, or at the Department of Linguistics, Stockholm University. Ten listeners participated in the Hold condition, and eight in the Change condition, with balanced numbers completing the $\mathrm{AB}$ and $\mathrm{BA}$ versions. Most participants completed the experiment in about 20 minutes, though some took longer due to listening to more stimuli multiple times.

\section{Results}

\subsection{Effect of question on responses}

Before exploring the prosodic data, we can observe a pattern that is consistent with other findings about perception of turn transition: namely, that participants were more likely to feel certain about their responses if they were asked to identify the version in which the speaker wanted to continue speaking rather than the version in which the speaker was done speaking $(\chi 2(1, \mathrm{~N}=1788)=37.4162, \mathrm{p}<0.001)$. Although speakers in the Change condition may have adopted the strategy of deciding which item sounded less like it would lead to continuation (one participant in the Change condition reported this strategy following the experiment), it appears that turn-hold is easier to identify, at least consciously, than turn 
ceding. The results discussed below are restricted to the cases in which participants reported being fairly sure about their responses.

\subsection{Length condition}

Consistent with the findings of Hjalmarsson \& Laskowski [13], the current study found an effect of lengthening on listeners' judgments about turn transition. Specifically, listeners in the Hold condition were more likely to choose Long items than Short items $(\chi 2(1, \mathrm{~N}=532)=4.841, \mathrm{p}<0.05)$, and listeners in the Change condition were more likely to choose Short items than Long ones $(\chi 2(1, \mathrm{~N}=320)=54.519, \mathrm{p}$ $<0.001)$.

\subsection{Pitch conditions}

For purposes of simplicity, only data from the Hold condition are reported here. Data in the Change condition showed similar (inverted) patterns, but further analysis is required to determine to what extent the difference in question impacted the pitch results.

Listeners heard pairs of stimuli which varied in their F0 peak height (Peak condition) and the location of the final F0 low (Truncation condition). Listeners in the Hold condition were more likely to choose tokens with higher pitch peaks and higher final F0 (assuming the same length). There was no statistically significant preference for the 8st version versus the 5st version compared to the 3st version in the Peak condition $(\chi 2(2, \mathrm{~N}=180)=3.8808, \mathrm{p}=0.1436)$. Similarly, there was no preference for greater degrees of truncation $(\chi 2(2, N=213)=1.1572, p=0.5607)$. Thus it appears that the difference is gradient, with higher pitch peaks and higher final pitch values sounding more continuation-like, regardless of

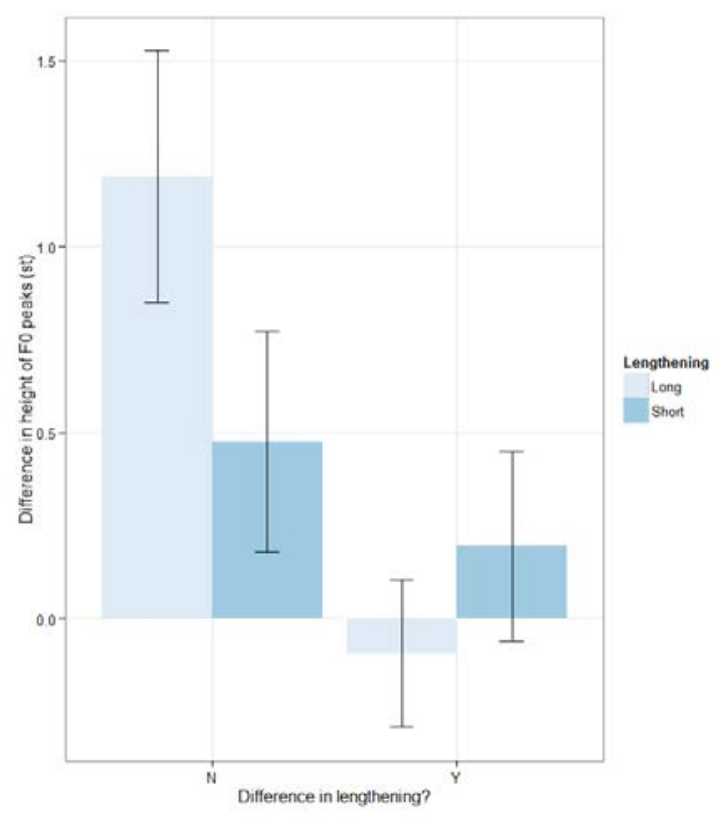

Figure 2: Difference in height of F0 peaks of stimuli identified as turn-holds compared to height of rejected stimuli. When there is a difference in lengthening between stimuli, there is no significant difference in F0 peak height, but when there is no difference in lengthening, higher F0 peaks are preferred. their value in an absolute sense.

If we treat the pitch values as continuous, rather than discrete, variables, we can use them in a statistical model with lengthening as a factor. A linear mixed model shows a main effect of the presence of a length difference, and the length of the chosen stimulus, in the prediction of the pitch height of the chosen turn in the Hold data: when stimulus length is different between the two stimuli, there is no difference in the mean pitch height across the chosen stimuli. However, when the two stimuli were the same length, there was a tendency for the chosen stimulus to have a higher pitch peak than the nonchosen stimulus. This is shown in Figure 2, with statistics in Table 1 (NB: the length of the chosen turn is included as a covariate in the model since a wider range of pitch variation was available in the Long than the Short items).

Table 1: Results of regression model: difference in pitch height between chosen and rejected stimuli when length differences are present or absent (Hold data). $* * *=p<0.001$

\begin{tabular}{|l|l|l|l|}
\hline Variable & B & SE(B) & t-value \\
\hline Length difference: $\mathrm{Y}$ & -0.7736 & 0.1841 & $-4.202^{* * *}$ \\
\hline \multicolumn{2}{|c|}{$\mathrm{R}^{2}=0.0993$ (Adj. $\left.\mathrm{R}^{2}=0.0976\right)$} \\
\hline
\end{tabular}

On the other hand, the degree of Truncation is not independently indicative of turn-hold when the height of the Peak is taken into account in the statistical model, as shown in Table 2.

Table 2: Results of regression model: difference in truncation between chosen and rejected stimuli when length differences are present or absent (Hold data), controlled for peak height.

\begin{tabular}{|l|l|l|l|}
\hline Variable & B & SE(B) & t-value \\
\hline Length difference: $\mathrm{Y}$ & -0.1614 & 0.2659 & -0.607 (ns) \\
\hline \multicolumn{2}{|l|}{$\mathrm{R}^{2}=0.1051$ (Adj. $\mathrm{R}^{2}=0.1034$ ) } \\
\hline
\end{tabular}

\section{Discussion}

The current study finds that final lengthening is likely to lead Swedish listeners to a turn-hold interpretation. If a lengthening cue is not available, increased height of the final (i.e. focal) pitch peak can also lead to a turn-hold interpretation. The final value to which pitch falls at the end of a turn also shows patterns of variation associated with turn-hold or turntransition, but truncation of the pitch contour does not appear to be a sufficient cue for turn-hold without the additional variation of the pitch peak height.

These data bring some clarity to previous reports about prosodic cues to turn hold and turn transition in Swedish. Previous studies on corpora found conflicting evidence about the role of final lengthening and pitch cues in turn transition in Swedish. This may in part arise from a problem of methodology: Hjalmarsson [11] and Hjalmarsson \& Laskowski [13] used prosodic data from the vicinity of silent pauses, which could have limited the sensitivity of those analyses to discover regularities in pitch and lengthening 
features, since silent pauses are not obligatory at potential turn boundaries. Consistent with [13], the current data support the conclusion that lengthening is a strong turn-hold cue for Swedish listeners, just as it is in English (cf. [5]).

Final lengthening can be found at prosodic boundaries across numerous languages, including in sign languages such as American Sign Language (ASL; Grosjean \& Lane [19]). As it appears to be a universal cue to prosodic structure, it may also be a universal cue to conversational functions such as turn-hold. Some possible evidence for this comes from research on ASL by Perlmutter [20]. Perlmutter argues that final lengthening in sign languages differs from final lengthening in other languages because it involves holding at the end of a gesture rather than lengthening the gesture itself. Articulatory holding gestures such as filled pauses or unreleased plosives are common at turn-hold locations in many languages (cf. e.g. [21], [22], [23]). If lengthening at possible turn ends can originate from a holding gesture similar to those found in ASL, the apparent discrepancy between lengthening as a boundary marker and lengthening as a continuation marker can be resolved.

Gussenhoven ([24]; [25]) argues that three Biological Codes underlie linguistic intonational systems, with intonational gestures originating in universal communicative factors and then being grammaticalized into linguistic systems, sometimes in different ways. This means that similar phonetic features can have different origins; high pitch can be associated both with greater arousal on the part of the speaker (Effort Code: higher pitch means that more energy has been invested into production) and with uncertainty or submissiveness (Frequency Code: higher pitch results from a smaller larynx, which is likely to be found in a comparatively small and/or weak creature). Final lengthening as a boundarymarking phenomenon, on one hand, could be treated as originating from the Production Code, with lengthening being a reflex of articulatory relaxation at the ends of units. Final lengthening as a turn-holding phenomenon, on the other hand, could originate from the Effort Code, with the extended production being the speaker's specific marking of his/her intention to continue speaking. The use of additional effort around turn-holds is also consistent with the findings in this study that turn-hold cues are considered by listeners to be more reliable than turn-transition cues. Sacks et al. ([1]), in their algorithm for how conversational participants determine who will speak next, give the priority at any turn-ending to a speaker other than the one whose turn has finished; an underlying default to transition between interlocutors is consistent with extra marking (prosodic, lexical or otherwise) being necessary in cases when a speaker wishes to hold the floor beyond a possible turn boundary.

Final lengthening was considered the most reliable cue for turn-hold by listeners. Since Central Swedish has a lexically contrastive pitch accent system, it was possible that there might be less flexibility in the availability of F0 variation for functions such as cueing turn-hold or turn-transition. However, the current study finds that Swedish listeners, when deprived of variation in final lengthening, will still make use of F0 variation in their judgments, with higher F0 peaks (or expanded final pitch range) being associated with turn-hold. This is initially a somewhat surprising finding, since expanded final pitch range has typically been associated with questions in Swedish, and might thus be expected to lead to turntransition, rather than turn-hold interpretations. That this does not occur in the current study has at least two possible explanations, which could be simultaneously true. First, the turns used as stimuli may have been difficult to interpret as questions, particularly the större and sågen items, which were statements depending on the speakers' knowledge. The instructions for the task might also have influenced listeners away from a question interpretation, since the stimuli were referred to as meningar (sentences).

A second possible explanation for the pitch effects observed is that the higher F0 peaks shifted the interpretation of the accents from broad focus to narrow focus. Gårding [7] points out that narrow focus can be enhanced when surrounding pitch accents are compressed. Although accents other than the final accents in the current stimuli were not manipulated, the expanded versions of the final accents (particularly the 8st case) may have meant that these accents were seen as expanded, or conversely, that other accents in the turns were compressed. Since narrow focus is often associated with new or otherwise prominent information, the turn-hold interpretation in these cases may not have had to do with pitch serving as a turn-holding cue, but rather with the sentencepragmatic interpretation that the speaker would have more to say about new or prominent information. This would also explain why pitch variation only had a statistically significant effect in cases when there was no variation in lengthening; listeners, not having true turn-hold cues available, would have had to resort to an alternative strategy in these cases.

The data reported here are only a subset of the data collected, with the important characteristic that they were cases in which listeners reported feeling fairly certain about their responses. It remains to be seen whether listeners' judgments in the unsure cases will follow similar patterns. If the pitch-related differences in judgments about the stimuli were indeed based on the focal structure of the sentences rather than directly turn-related cues, the results may differ in cases where participants might not have had conscious access to the differences in interpretation.

\section{Conclusions}

The current study has demonstrated that Swedish listeners make use of both final lengthening and pitch variation in their judgments about whether speakers wish to hold the floor or allow someone else to speak. Furthermore, listeners were more confident in their judgments about turn-hold rather than turntransition, suggesting an asymmetry between these two actions in interaction. The difference in accessibility of the lengthening and pitch cues suggests a priority for lengthening, with pitch possibly leading to other pragmatic interpretations which are only used when other cues are not available. More research is necessary to clarify the interaction between these different kinds of variation.

\section{Acknowledgements}

I am very grateful to David House for guidance on the experimental design, to Anna Hjalmarsson for providing me with her stimuli, to Jana Götze, Mattias Heldner and Myra Öberg for assistance with recruiting participants, and to Niklas Vanhainen for proofreading my Swedish. This research was supported by the postdoctoral grant "Perception of prosody in linguistic contexts" from the Swedish Science Foundation (Vetenskapsrådet). 


\section{References}

[1] Sacks, H., Schegloff, E.A. \& Jefferson, G. (1974) A simplest systematics for the organisation of turn-taking for conversation. Language 50(4): 696-735.

[2] Heldner, M. \& Edlund, J. (2010) Pauses, gaps and overlaps in conversation. Journal of Phonetics 38: 555-568.

[3] Heldner, M. (2011) Detection thresholds for gaps, overlaps, and no-gap-no-overlaps. Journal of the Acoustical Society of America 130(1): 508-513.

[4] Local, J.K., Kelly, J. \& Wells, W.H.G. (1986) Towards a phonology for conversation: turn-taking in Tyneside English. Journal of Linguistics 22: 411-437.

[5] Gravano, A. \& Hirschberg, J. (2009) Turn-yielding cues in taskoriented dialogue. Proceedings of SIGDIAL 2009, Queen Mary University of London, UK, 253-261.

[6] Auer, P. (1996) On the prosody and syntax of turn-continuations. In Couper-Kuhlen, E. \& Selting, M. (eds.) Prosody in conversation: interactional studies. Cambridge, UK: Cambridge University Press, 57-100.

[7] Gårding, E. (1989) Intonation in Swedish. Lund University Department of Linguistics Working Papers 35: 63-88.

[8] Gussenhoven, C. \& Bruce, G. (1999). Word prosody and intonation. In van der Hulst, H. (Ed.) Word prosodic systems in the languages of Europe. Berlin/New York: de Gruyter, 233271.

[9] House, D. (2004) Final rises and Swedish question intonation. In Proceedings of FONETIK 2004, Stockholm University, Sweden, pp. 56-59.

[10] House, D. (2006) Perception and production of phrase-final intonation in Swedish questions. In Bruce, G., \& Horne, M. (Eds.), Nordic Prosody, Proceedings of the IXth Conference, Lund 2004. Frankfurt am Main: Peter Lang, 127-136.

[11] Hjalmarsson, A. (2011) The additive effect of turn-taking cues in human and synthetic voice. Speech Communication 53: 23-25.

[12] Edlund, J. \& Heldner, M. (2005) Exploring prosody in interaction control. Phonetica 62: 215-226.

[13] Hjalmarsson, A. \& Laskowski, K. (2011) Measuring final lengthening for speaker-change prediction. In Proceedings of $12^{\text {th }}$ Interspeech, Florence, Italy.

[14] Wichmann, A. \& Caspers, J. (2001) Melodic cues to turn-taking in English: evidence from perception. In Proceedings of SIGDIAL, Aalborg, Denmark.

[15] Ford, C.E., Fox, B.A., \& Thompson, S.A. (1996) Practices in the construction of turns: the "TCU" revisited. Pragmatics 6(3): 427-454.

[16] Hjalmarsson, A., Wik, P. \& Brusk, J. (2007) Dealing with DEAL: a dialogue system for conversation training. In Proceedings of SIGDIAL, Antwerp, Belgium, 132-135.

[17] Boersma, P. \& D. Weenink (2013). Praat: doing phonetics by computer [Computer program]. Available http://www.praat.org/

[18] Xu, Y. \& Sun, X. (2002) Maximum speed of pitch change and how it may relate to speech. Journal of the Acoustical Society of America 111: 1399-1413.

[19] Grosjean, F. \& Lane, H. (1977) Pauses and syntax in American Sign Language. Cognition 5: 101-117.

[20] Perlmutter, D. (1993) Sonority and syllable structure in American Sign Language. In Coulter, G.R. (Ed.) Current issues in ASL phonology. New York: Academic Press, 227-261.

[21] Local, J. \& Kelly, J. (1986) Projection and silences: notes on phonetic and conversational structure. Human Studies 9: 185204.

[22] Ogden, R. (2001) Turn transition, creak and glottal stop in Finnish talk-in-interation. Journal of the International Phonetic Association 31: 139-152.

[23] Jasperson, R. (2002) Some linguistic aspects of closure cut-off In Ford C. E., Fox B. A. \& Thompson S. A. (Eds.), The language of turn and sequence. New York: Oxford University Press, 257286.

[24] Gussenhoven, C. (2002) Intonation and interpretation: phonetics and phonology. In Proceedings of Speech Prosody 2002, Aix-enProvence, France, 47-58.
[25] Gussenhoven, C. (2004) The phonology of tone and intonation. Cambridge, UK: Cambridge University Press. 